\title{
An Analysis of the Performance of Smoke Alarms
}

\author{
THOMAS G. CLEARY \\ Fire Research Division, Engineering Laboratory \\ National Institute of Standards and Technology \\ Gaithersburg, MD 20899 USA
}

\begin{abstract}
Test results from the NIST 2008 Smoke Alarm Sensitivity Study were used in a smoke alarm performance analysis to examine the effects of pre-movement time, reduced travel speeds through smoke, and smoke optical density limit on occupant survivability given different smoke alarm installations. Smoke alarm installations that meet the requirements in the current National Fire Alarm Code NFPA 72 were considered. Alarm times from commercially-available photoelectric, ionization, and dual photoelectric/ionization alarms were used in the analysis to examine the effects of smoke alarm type on the predicted survivability for a range of fire and egress scenarios. Fire scenarios included both flaming and initially smoldering upholstered chair mock-ups. Egress scenarios considered occupants located in, or remote from the room of fire origin. Reduced travel speed through smoke was included in the analysis. Prior to occupant movement and as an occupant travels to the exit, the fractional effective dose from toxic gas and heat exposure were computed to determine survivability. The concept of relative effectiveness as performance metric for smoke alarms is introduced. The relative effectiveness is the fraction of occupants that successfully escape a given fire and egress scenario. It is computed by considering a frequency distribution for the premovement time and determining the cumulative fraction of occupants that successfully escape. Thus, the relative effectiveness of a smoke alarm type or installation requirement can be averaged over a large number of fire and egress scenarios. The pre-movement frequency distribution was modeled as a lognormal function. Experimental studies suggest that the median value of the distribution relates to characteristics of the population and a geometric standard deviation of 1.6 characterizes the width the distributions. The distribution median was varied to examine relative effectiveness skewed to more vulnerable populations (those slower to react). Travel speed was modeled as a function of smoke optical density which predicts reduced travel speed as thicker smoke is encountered. Model results showed photoelectric alarms had the lowest relative effectiveness values for flaming fires, while ionization alarms had the lowest relative effectiveness values for smoldering fires. These trends were expected based the results of previous studies. It was observed that there can be a steep increase in relative effectiveness, depending on the smoke alarm type and fire scenario, as the smoke optical density limit was increased from $0.25 \mathrm{~m}^{-1}$ to $0.50 \mathrm{~m}^{-1}$. However, the ranking of smoke alarms tend to remain the same. Given the magnitude of statistically significant mean values of relative effectiveness for all flaming and smoldering fires considered, the model results suggest that there is a benefit from a combination of alarm technologies, and that vulnerable populations who may require significantly more time to escape, regardless of the fire scenario, would benefit the most from dual alarms or side-by-side photoelectric and ionization alarms.
\end{abstract}

KEYWORDS: detection, egress, smoke, toxicity, risk assessment.

\section{INTRODUCTION}

Experimental studies have shown that smoke alarms provide valuable early warning time for occupants exposed to fire in residential settings [1-4]. Fire safety codes and standards in many jurisdictions have mandated smoke alarms to enhance life safety in home fires. In the US, building and fire codes refer to the National Fire and Signaling Alarm Code, NFPA 72 [5], for smoke alarm minimum requirements in residential occupancies. These requirements rely on judgments of effectiveness for a given smoke alarm placement strategy. Over the last three decades, smoke alarm requirements have gone from one alarm per household, to one alarm on every level and interconnected, to interconnected alarms on every level and in every bedroom. Interconnected alarms provide a signal path from one alarm to another such that if any smoke alarm activates, all alarms activate. Individual alarm sensitivity requirements as specified by ANSI/UL 217 have not substantially changed over the last three decades, nor have the alarm sensing technologies, photoelectric and ionization. Combination alarms that contain both photoelectric and ionization sensors have been available for over ten years, and represent an effort to achieve better overall performance for both flaming and smoldering fire detection. 
Although the aforementioned studies have shown that either current photoelectric or ionization technologies provide sufficient warning for a wide range of fire scenarios, new technologies require performance assessments relative to current accepted technologies and placement requirements. A relative performance assessment of smoke alarm technologies and placement strategies should quantify effectiveness over a range of fire and occupant egress scenarios so that results are not biased toward a particular fire or occupant egress scenario, but designed to represent expected performance ranking (higher, equivalent, or lower) in residential fires.

Performance assessments of smoke alarms have focused on estimating whether or not occupants will have sufficient time to egress a residence when initially alerted to a fire by a smoke alarm [1-4]. The available safe egress time (ASET) is defined as the time increment starting from the time when an occupant is alerted to a fire (usually taken as the alarm activation time) to the time the occupant has been exposed to an incapacitating dose (toxic gas or heat) or a limiting smoke obscuration. The time increment it takes to egress a given residence starting from the time an occupant is alerted to a fire to the time the occupant leaves the residence is defined as the required safe egress time (RSET). (ISO 13571 [7] defines both ASET and RSET as starting from the time of ignition of the fire.) Thus, if ASET is greater or equal to RSET, then it is deemed that occupants would have had sufficient time to egress. A "margin of safety" has been proposed as a performance metric and defined as the difference between ASET and RSET that have positive values [6]. Therefore, the margin of safety is a function of a particular fire scenario, incapacitation levels and smoke limit chosen, egress path and assumptions about occupant behavior, and the actual performance of a given smoke alarm. Since performance assessments involve a multitude of fire and egress scenarios it is not clear how to aggregate each individual margin of safety, or how to account for scenarios where ASET is less than RSET.

In order to determine ASET, the time it takes to reach some limiting value of toxic gas or heat exposure or smoke obscuration is needed. The standard ISO 13571 Life Threat from Fires - Guidance on the Estimation of Time Available for Escape Using Fire Data [7] provides a methodology for estimating available safe egress time from smoke, heat, and toxic gas exposure. Heat and toxic gas exposure effects are cumulative with threshold values of incapacitation for exposed persons. The toxic gas model, and the heat and radiative energy model specified in the ISO standard [7] use equations that integrate doses of relevant gases or heat exposure into a value termed the fractional effective dose (FED). The equations are available in the standard [7], or in the NIST Home Smoke Alarm Report [2]. A height of $1.5 \mathrm{~m}$ (5 ft) from the floor is typically chosen as the sampling location to evaluate toxic gas, heat exposure, and smoke obscuration as this approximates the head height of a standing person. A fractional effective dose of 1.0 corresponds to the dose where one half of the population is expected to become incapacitated. A fractional effective dose of 0.3 corresponds to an incapacitation threshold for more susceptible people (this value was used in the NIST study [2]). While the ISO standard suggests a distribution where a FED of 0.3 would represent the threshold for about $11 \%$ of the population, there is no data in which to support this.

Smoke exposure limit is a threshold smoke concentration or optical density where visibility along escape routes is significantly reduced. At a smoke optical density where an occupant would feel the need to stop attempting to egress and retreat, or become so disoriented that they no longer make any significant progress towards an exit is considered the end point in the ASET evaluation. The smoke optical density limit specified in the NIST home smoke alarm tests was an optical density (OD) value of $0.25 \mathrm{~m}^{-1}$ [2], while ISO 13571 proposes an OD of $3.4 \mathrm{~m}^{-1}$ [7], a value where it was estimated that one could not see more than an arm's length $(0.5 \mathrm{~m})$ distance. Experimental studies have shown that smoke obscuration tends to reduce the walking speed of people moving through a smoke-filled space [8]. This observation supports the contention that sufficient smoke concentration impedes egress.

In previous studies [2-4], a maximum RSET for residential settings was chosen as a limiting value where most occupants should have sufficient time to egress based on average pre-movement times (the time spent on tasks not associated with moving to alert others or toward the exit, like waking and recognizing the there may be a fire, assessing the situation, gathering personal belongings, waking others, etc.) and average walking speeds which works out to be about two minutes. Nober et al. [9] measured the time it took sleeping occupant to awaken to an alarm and egress their homes. They found most occupants would exit homes in $90 \mathrm{~s}$. In a study by Proulx et al. [10] it was suggested that the maximum RSET may be longer than 11 min with a minimum RSET of one minute. If two potential occupant activities accounted for in 
their maximum RSET are ignored (fighting the fire, and dressing for winter conditions, since they are counterproductive to a rapid egress), the maximum is reduced to $220 \mathrm{~s}$, which is still almost double previous estimates and observations. It is not difficult to surmise that only a very small fraction of occupants capable of escaping (not including occupants who do not wake up or are severely mobility impaired) might take an extra $100 \mathrm{~s}$ beyond $120 \mathrm{~s}$ to egress. However, the margin of safety would decrease by $100 \mathrm{~s}$, and for fast growing flaming fires, it is possible no alarm would provide any margin of safety. One way to account for such disparate values in maximum RSET is to assign a distribution to the time taken for pre-movement activities. Thus, RSET is not quantified by an arbitrary maximum, but by a frequency distribution. Purser [11] has suggested pre-movement time distributions be categorized in terms of design behavioral scenarios, of which one is sleeping, familiar with surroundings (e.g., residents in an apartment or house.) There are limited experimental observations in the literature that provide quantification of pre-movement distributions for residential occupancies $[9,10,12]$.

While the travel speed itself could be represented by a distribution, normally a mean value is employed for distinct populations (i.e., young adult or elderly) since the range in travel speed typically does not translate into a large change in RSET. Travel time can be evaluated in a deterministic manner if travel routes are pre-determined and travel speed is defined. A representative travel time can be determined with a fixed travel distance and travel speed for a given scenario. The NIST Home Smoke Alarm Study determined travel times in such a manner. An alternative to a constant travel speed is to let the speed be a function of the local smoke concentration (e.g., Purser [11] and Akizuki et al. [13].) This imposes a penalty on travel speed based on the optical density an occupant is traversing through. The assignment of a reduced travel speed through smoke accounts for some of the negative impact of reduced visibility. Furthermore, since smoke obscuration tends to increase as a fire progresses, an increase in pre-movement time will cause an increase in travel time for a particular scenario. Thus, a tenability criterion is coupled to the egress performance as suggested by Gwynne and Rosenbaum [14] to counterbalance assumptions made in selecting tenability limits.

\section{RELATIVE EFFECTIVENESS MODEL}

A model was designed to estimate the relative effectiveness of different types of smoke alarms in residential settings. Given a particular fire and egress scenario, a successful outcome is produced when an occupant being considered escapes the home through a normal exit. That is, the occupant exposure does not reach the limiting FED for toxic gases or heat, and the local smoke optical density is below the limiting value as the occupant traverses the egress path. The model integrates an occupant's toxic gas and heat exposure, and tracks smoke optical density and travel progress until an exit is reached over a range of incremented pre-movement times. Relative effectiveness is determined by summing the areas under the (normalized) frequency distribution curve for increments of pre-movement time where an occupant successfully escapes. Thus, relative effectiveness is presented as a fraction of successful escape attempts. Individual fractions of successful escape attempts for specific scenarios can be averaged to yield an overall effectiveness value. Thus results from a range of egress scenarios and fire scenarios can be combined to produce a single relative effectiveness value that represents a performance ranking of a particular alarm and placement strategy in residential fires.

\section{Pre-movement Time Distribution}

The pre-movement time for residential fire scenarios is usually a large fraction of the total time it takes to egress. Sample pre-movement activities identified in the NIST Home Smoke Alarm Report suggest an average time of $55 \mathrm{~s}$ for a young couple to dress, call the fire department, gather personal belongings and awaken two children, and $80 \mathrm{~s}$ for an elderly couple, with egress times of $90 \mathrm{~s}$ and $135 \mathrm{~s}$ respectively [2]. Nober et al. [9] conducted household field experiments of evacuation times when awakened by smoke alarms. Subjects were instructed to turn on a light, awaken others and egress as quickly as possible. For 40 households that had adult populations between 20-50 years of age, the mean evacuation time was about $48 \mathrm{~s}$, with 30 of 40 households evacuated in less than one minute, and all households evacuated in less than two minutes. For 20 households with elderly occupants between the ages of 63-75 years of age, the mean evacuation time was about $66 \mathrm{~s}$, with 8 of 20 households evacuating in less than one minute, and all but one household evacuating in less than $90 \mathrm{~s}$. Some of the spread in the observed evacuation times is most likely attributed to variation in individual pre-movement times. 
Experimental studies have been conducted to determine the time it takes people to complete certain tasks upon being alerted by a smoke alarm. Nober et al. [9] measured the time it took college-aged subjects to shut off the "smoke alarm" (a tape recorder playing a smoke alarm sound), and phone the local fire department. The first experiment characterizes awakening/recognition time and a very short travel time to shut off the alarm. The second experiment adds a more complex pre-movement activity, making a phone call. The results for these two experiments are presented as histograms in Figs. 1 and 2. Plotted with the histograms are normalized frequency distributions in the form of log-normal functions characterized by the experimental median time $9 \mathrm{~s}$ and $55.5 \mathrm{~s}$, and a geometric standard deviation of 1.6. Duncan [12] conducted experiments to measure the time it took subjects to de-activate a smoke alarm programmed to alarm at random times between $6 \mathrm{pm}$ and 6 am and located in a hallway outside of a bedroom in various homes. The subjects included primarily college-aged students $(65 \%)$, a population that had no smoke alarms previously installed in there homes $(25 \%)$, and elderly subjects $(10 \%)$. A histogram of the results for all populations is presented in Fig. 3. A log-normal distribution is also shown using the median experimental time (16 s) and a geometric standard deviation of 1.6.

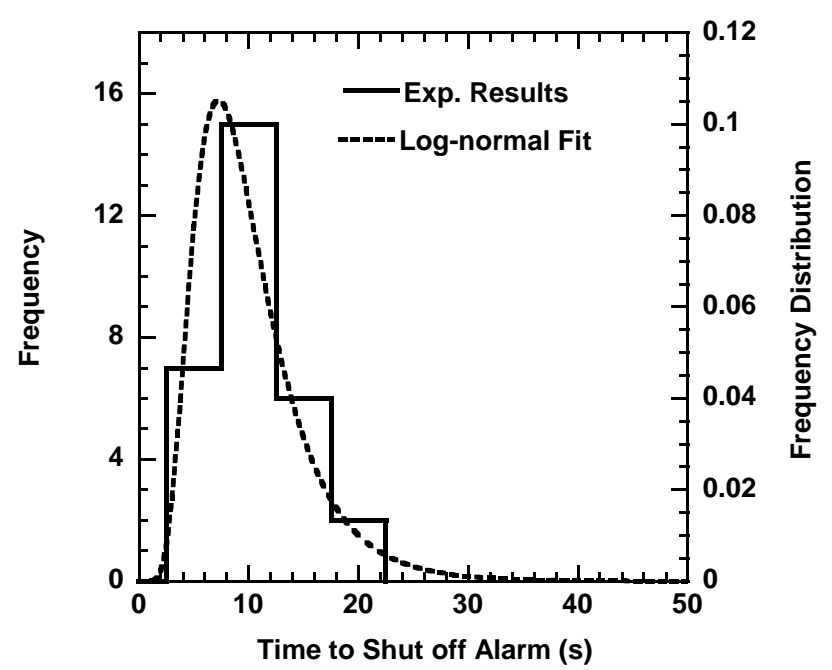

Fig. 1. Time college-aged subjects took to shut off an alarm after awakening.

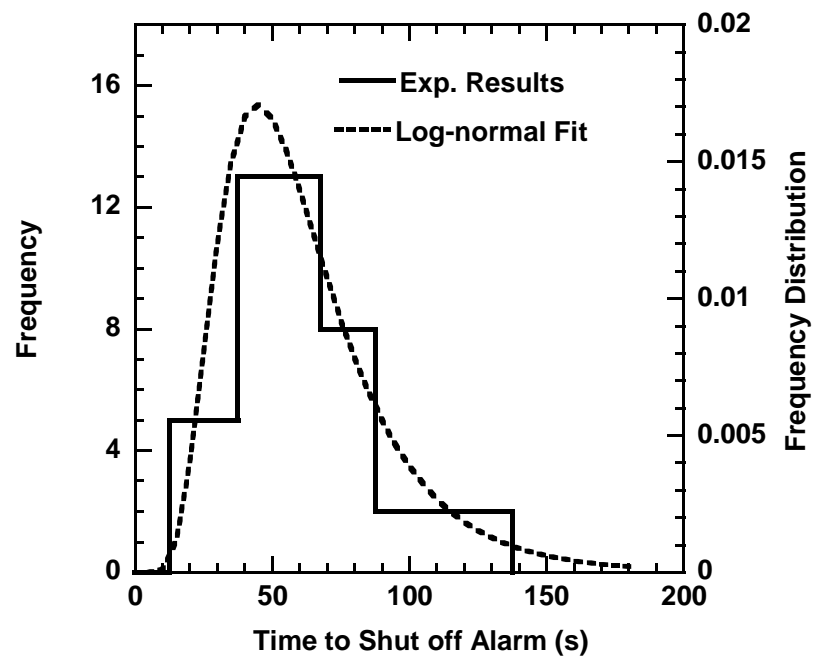

Fig. 2. Time college-aged subjects took to shut off an alarm and phone the fire department after awakening. 


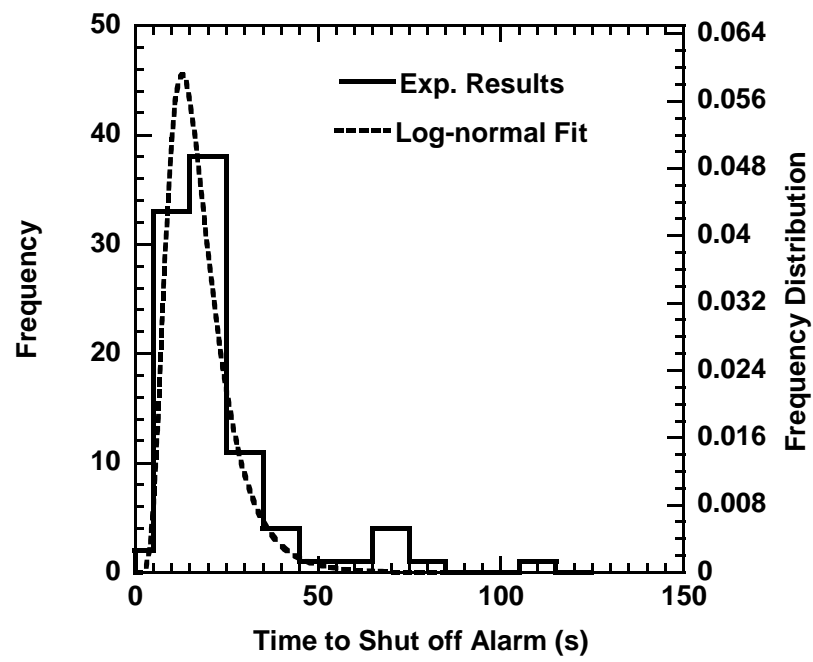

Fig. 3. Time subjects took to shut off an alarm placed in a hallway.

The data from all three sets of experiments quantify primarily pre-movement times and can be characterized by log-normal distributions having a geometric standard deviation $\left(\sigma_{g}\right)$ of 1.6. The median time appears to be a function of the pre-movement activities and characteristics of the population. While these studies present a limited picture of the frequency distribution of pre-movement time, they do suggest a functional form and a nominal width of the distributions one might expect. It is reasonable to expect different populations to have a different median pre-movement time based on age, mobility, cultural norms, impairment, etc.; therefore, one could examine alarm effectiveness for more vulnerable populations by increasing the median pre-movement time.

In the model calculations, three log-normal distributions representing assumed pre-movement time frequency distributions were considered: a median time of $16 \mathrm{~s}$ and a geometric standard deviation of 1.6 representing an easily alerted, mobile population, a median time of $35 \mathrm{~s}$ and a geometric standard deviation of 1.6 representing a population slower to respond, (e.g. prone to investigate or pre-occupied with gathering personal effects) and a median time of $55 \mathrm{~s}$ and a geometric standard deviation of 1.6 representing the slowest population, i.e., the elderly, or partially impaired. The three distributions are shown in Fig. 4.

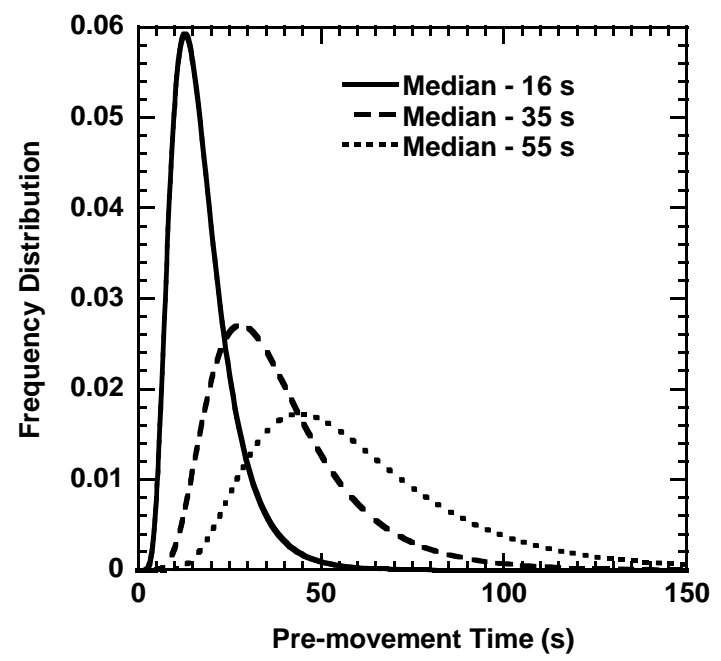

Fig. 4. Normalized log-normal frequency distributions of pre-movement time. 


\section{Travel Speed}

The evacuation studies of Nober et al. [9] were conducted with no smoke or fire present for obvious safety reasons. The Home Smoke Alarm Study [2] egress time estimates were based on normal travel speeds uninhibited by smoke, and it was noted in the report that no feedback between the fire and the occupants was accounted for in the escape times. In order to properly assess some of the effects a distribution of premovement times has on RSET, evacuation should be affected by changing environmental conditions. An obvious choice is the effect of decreased travel speed through smoke which has been investigated by Jin and Yamada [8]. They measured subject walking speeds down a smoke filled corridor using a highly irritating smoke from burning wood cribs, and a much less irritating smoke from burning kerosene. The decrease in walking speed for a fixed smoke optical density was greater for the irritating wood smoke. Additional experiments were conducted using the burning wood crib smoke and subjects with their mouths and noses covered with a multi-layered towel yielded walking speeds closer to the non-irritating smoke results. Since smoke alarm studies are not limited to burning wood cribs or kerosene, it seems reasonable to use an intermediate value of travel speed for a given optical density. Figure 5 shows a curve that represents an intermediate estimate of travel speed through smoke. The points are values from curves that Jin and Yamada [8] used to represent an average for each type of smoke. Two features of the intermediate estimate curve are that it starts with a walking speed of $1.00 \mathrm{~m} / \mathrm{s}$ and it asymptotically approaches $0.095 \mathrm{~m} / \mathrm{s}$. Thus in any smoke concentration, walking speed doesn't drop below $0.095 \mathrm{~m} / \mathrm{s}$. While there are no data to support this minimum walking speed, it is included to allow for the computation of egress times at high optical densities with a significant speed penalty. However, the ASET smoke optical density limit is always enforced. If the optical density limit is reached, an occupant is assumed to stop moving or retreat and shelter, which means they do not successfully escape.

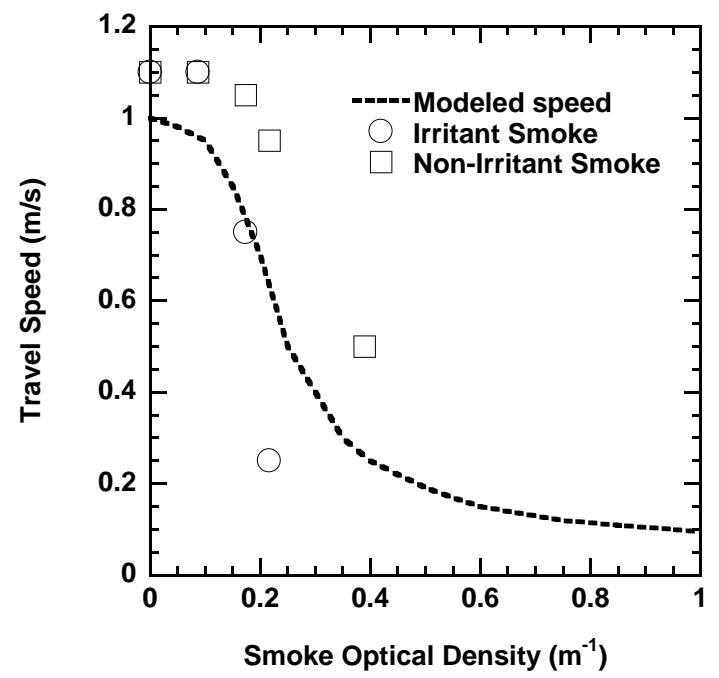

Fig. 5. Travel speed as a function of optical density for different smoke conditions.

\section{Model Equations}

The alarm times for a given configuration of alarm type(s) and locations for every fire scenario are determining values for relative effectiveness. Escape time $(E T)$ for a fixed pre-movement time, $t_{p}$, is computed using Eq. 1 where $t_{\text {alarm }}$ is the alarm time, and $t_{\text {travel }}$ is the travel time it takes to traverse the predetermined path to the exit.

$$
E T=t_{p}+t_{\text {travel }}+t_{\text {alarm }}
$$

Equation 2 is used to estimate the travel time from the sum of times it takes to traverse $k$ separate travel segments. While traveling through a space where the smoke concentration is varying, the time it will take 
to walk a fixed distance is approximated by summing the incremental distance traveled every second using the average walking speed $\left(S_{i}\right)$ over that time increment $(\delta t)$ up to a time $\left(t_{j}\right)$ where the sum is equal to or greater than the fixed distance $\left(L_{j}\right)$ for each travel segment.

$t_{\text {travel }}=\sum_{j=1}^{k} t_{j}$, where $t_{j}$ is defined by $L_{j} \leq \sum_{i=1}^{t_{j}} S_{i} \delta t$

The maximum pre-movement time where an escape attempt is successful is identified by incrementing the pre-movement time starting from alarm initiation (pre-movement time $=0$ ) and comparing the escape time to the time to reach a limiting threshold for toxic gas, heat, or smoke optical density (ASET). Once the premovement time is incremented to a value where a limiting threshold is reached prior to escape, the previous pre-movement time is deemed the maximum successful pre-movement time, $t_{p, \max }$.

$$
A S E T>t_{p, \max +1}+t_{\text {travel }}+t_{\text {alarm }}
$$

Integrating the normalized pre-movement frequency distribution from 0 to $t_{p, \max }$ yields the fraction of successful escapes $\left(\mathrm{X}_{s}\right)$ for a population characterized by the median pre-movement time $\left(t_{\text {median }}\right)$ and geometric standard deviation $\left(\sigma_{g}\right)$.

$$
\mathrm{X}_{s}=\int_{0}^{t_{p, \max }} \frac{1}{\sqrt{2 \pi} t_{p} \ln \sigma_{g}} \exp \left(-\frac{\left(\ln t_{p}-\ln t_{\text {median }}\right)^{2}}{2\left(\ln \sigma_{g}\right)^{2}}\right) d t_{p}
$$

The arithmetic average of the individual fraction of successful escapes $\left(\mathrm{X}_{s}\right)$ over all $n$ egress scenarios considered is the fraction of successful escapes for a given fire scenario $\left(\mathrm{X}_{f}\right)$.

$\mathrm{X}_{f}=\frac{\sum_{i=1}^{n} \mathrm{X}_{s}(i)}{n}$

The relative effectiveness $\left(\mathrm{E}_{\mathrm{r}}\right)$ is the arithmetic average of the individual fraction of successful escapes for all $m$ fire scenarios.

$E_{r}=\frac{\sum_{i=1}^{m} \mathrm{X}_{f}(i)}{m}$

\section{Fire and Egress Scenarios}

The experimental dataset used to evaluate the relative effectiveness of smoke alarms is the data from the NIST Smoke Alarm Sensitivity Study [15]. The dataset included 12 initially flaming (four scenarios and three replicates) and 12 initially smoldering fires (four scenarios and three replicates) conducted in a mockup of a small house or apartment. Commercially available smoke alarms including photoelectric, ionization and two dual alarms from different manufacturers were used in the study. Details on the experimental measurements, and alarm times are given in Ref. [15].

A floor plan showing the layout and an exit door is shown in Fig. 6. The exit door and additional bedroom were not constructed, but were defined for the egress scenarios. Travel distances for each travel path (TP) (master bedroom, living room, hallways, and bedroom) are indicated on the floor plan. Time to alarm from alarm sets at locations S3 and S6 were used in the analysis. Some assumptions were made to provide a complete dataset for analysis. First, the bedroom added to the floor plan was assumed to remain free of 
smoke, elevated temperature, and toxic gases because the door is assumed to be closed most of the time. Second, the carbon monoxide and carbon dioxide concentrations in the living room space were taken as an average of the measured values in each hallway location. Third, for tests where smoke concentration values were not available for a particular location, the nearest measured value was used.

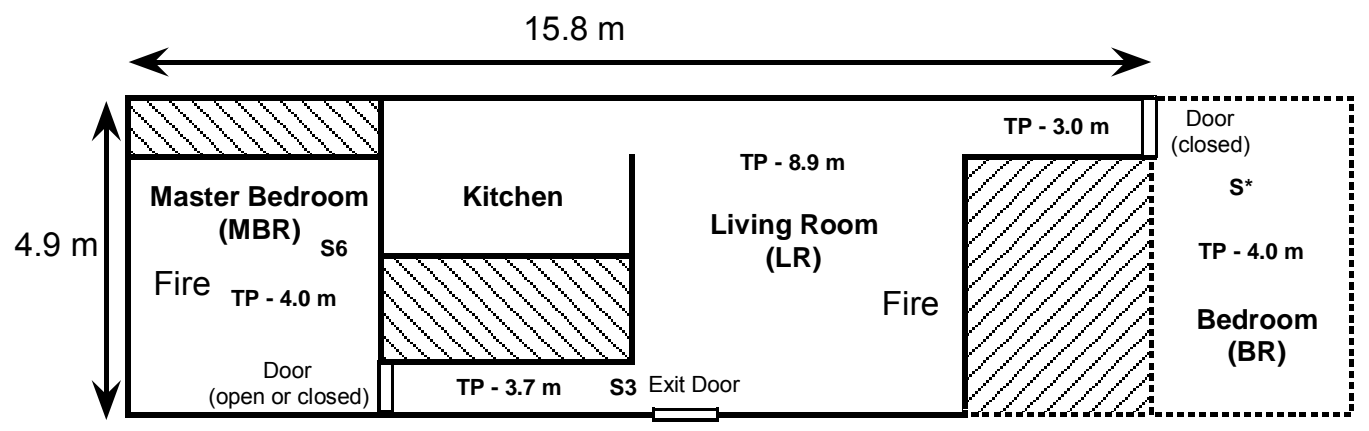

Fig. 6. Floor plan showing the layout and exit door.

Thirteen egress scenarios were included in the analysis, and they are detailed in Table 1 . They contain both direct egress paths and backtracking paths to account for investigation and/or alerting other occupants. The travel path for bedrooms is defined as $4.0 \mathrm{~m}$ for the initial exit, or subsequent entry then exit. Depending on the fire location, some scenarios consider occupants initially located in the room of fire origin.

Table 1. Travel paths, and distance traveled for each egress scenario considered.

\begin{tabular}{|l|l|c|c|}
\hline Scenario & Travel path (TP) & Travel segments & Travel distance (m) \\
\hline 1 & MBR - Exit & 2 & 7.7 \\
\hline 2 & LR - Exit & 1 & 8.9 \\
\hline 3 & BR - Exit & 3 & 15.9 \\
\hline 4 & MBR - BR - Exit & 7 & 35.5 \\
\hline 5 & BR - MBR - Exit & 6 & 27.3 \\
\hline 6 & LR - BR - Exit & 5 & 27.8 \\
\hline 7 & LR - MBR - Exit & 4 & 20.3 \\
\hline 8 & LR - BR - MBR - Exit & 8 & 39.2 \\
\hline 9 & LR - MBR - BR - Exit & 9 & 48.1 \\
\hline 10 & MBR - LR - MBR - Exit & 6 & 28.0 \\
\hline 11 & BR - LR - BR - Exit & 7 & 34.8 \\
\hline 12 & MBR - BR - MBR - Exit & 10 & 46.9 \\
\hline 13 & BR - MBR - BR - Exit & 11 & 55.1 \\
\hline
\end{tabular}

The time to alarm for any experimental test and alarm type was the first alarm to activate at location S3 or S6 of the particular type being examined. The assumption was made that alarms located at S3, S6 and in the additional bedroom were interconnected, thus when the first alarm activated, all alarms activated.

\section{RESULTS AND DISCUSSION}

Escape times for 37 pre-movement times from 0 to $180 \mathrm{~s}$ in $5 \mathrm{~s}$ interval were computed for every egress scenario, every test, and every alarm type. Besides the pre-movement distributions, four different smoke optical densities were specified as the smoke tenability limit to examine the sensitivity of this value on the relative effectiveness. The values were: $0.25 \mathrm{~m}^{-1}$, (the value used in the NIST study [2]), $0.5 \mathrm{~m}^{-1}, 1.0 \mathrm{~m}^{-1}$, and $1.7 \mathrm{~m}^{-1}$ (half the value suggested in the ISO standard [7]).

Figure 7 shows the time to escape for a test with a flaming upholstered chair in the living room and egress Scenario 8 (Table 1). Results are presented as a function of the pre-movement time for each smoke alarm initial response. For lower values of the pre-movement time an increment in pre-movement time translates 
into an equal increment in escape time. The increasing slope of the curve passing through the escape times indicates escape time increases faster for a given pre-movement time increment as the occupant has to travel through an ever-increasing smoke concentration. The results for a given egress scenario, test dataset, and alarm type being considered reduce to the largest pre-movement time where the outcome is a successful escape through the exit. Toxic gas and heat FED values of 0.3 were specified.

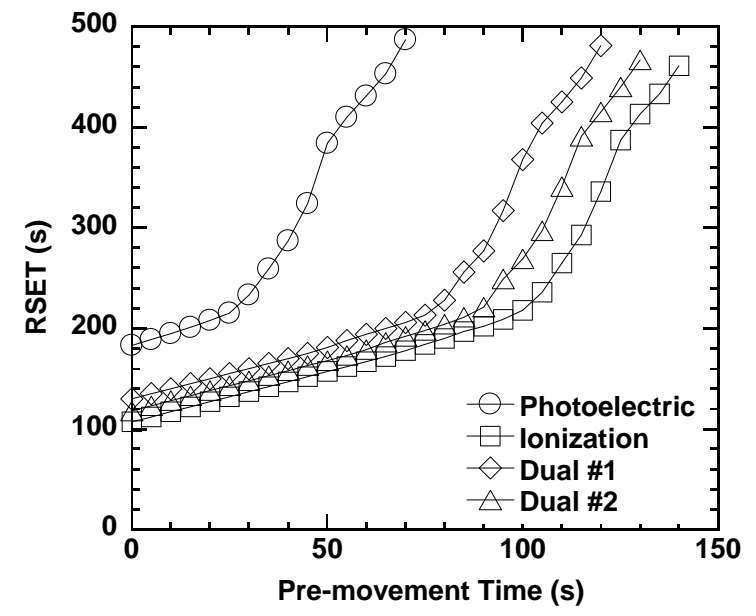

Fig. 7. Escape time as a function of pre-movement time for Scenario 8 with a flaming chair in the living room.

Table 2 shows the maximum pre-movement times yielding successful escape for the different smoke optical density limits. Considering the more vulnerable, slowest population, the cumulative fraction of the pre-movement distribution that successfully escape $\left(\mathrm{X}_{s}\right)$ varied from $0.02,0.88,0.75$, and 0.83 assuming an optical density limit of $0.25 \mathrm{~m}^{-1}$, and $0.26,0.94,0.86$, and 0.90 assuming an optical density limit of $1.7 \mathrm{~m}^{-1}$ for P1, I1, D1, and D2 respectively. The average relative effectiveness for every egress scenario, and every test replicate represents the relative effectiveness $\left(\mathrm{X}_{f}\right)$ for a given fire scenario, tenability limits and premovement distribution.

Table 2. Maximum pre-movement time that allows successful escape for Scenario 8 with a flaming chair in the living room.

\begin{tabular}{|c|c|c|c|c|}
\hline $\begin{array}{c}\text { Optical density } \\
\left.\text { limit } \mathbf{( m}^{-\mathbf{1}}\right)\end{array}$ & $\begin{array}{c}\text { Photoelectric } \\
\text { alarm P1 (s) }\end{array}$ & $\begin{array}{c}\text { Ionization alarm } \\
\mathbf{I 1}(\mathbf{s})\end{array}$ & $\begin{array}{c}\text { Dual alarm } \\
\text { D1 (s) }\end{array}$ & $\begin{array}{c}\text { Dual alarm } \\
\text { D2 (s) }\end{array}$ \\
\hline 0.25 & 20 & 95 & 75 & 85 \\
\hline 0.50 & 30 & 105 & 80 & 95 \\
\hline 1.00 & 35 & 110 & 85 & 95 \\
\hline 1.70 & 40 & 115 & 90 & 100 \\
\hline
\end{tabular}

Figures 8-10 show the fraction of successful escapes $\left(\mathrm{X}_{f}\right)$ for a flaming fire scenario as a function of alarm type and optical density limit for each frequency distribution. The flaming fire scenario was an upholstered chair in the living room. For every frequency distribution and optical density limit, the relative effectiveness of the photoelectric alarm was lower than ionization or dual alarms. This is expected since ionization sensors tend to alarm sooner to flaming fires than photoelectric sensors. There was a sharper increase in fraction of successful escapes from an optical density limit of $0.25 \mathrm{~m}^{-1}$ to $0.50 \mathrm{~m}^{-1}$, than from $0.5 \mathrm{~m}^{-1}$ to $1.7 \mathrm{~m}^{-1}$ for photoelectric alarms with every frequency distribution. 


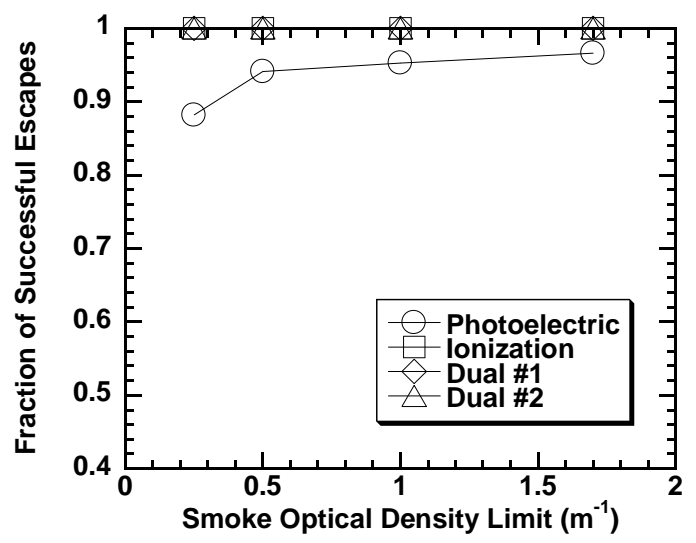

Fig. 8. Fraction of successful escapes $\left(\mathrm{X}_{f}\right)$ for the living room flaming chair fire considering the easily alerted, mobile group.

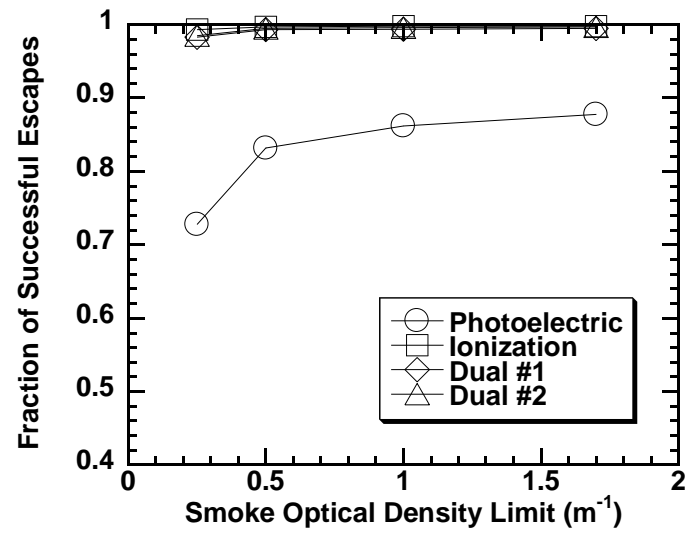

Fig. 9. Fraction of successful escapes $\left(\mathrm{X}_{f}\right)$ for the living room flaming chair fire considering the moderately mobile group.

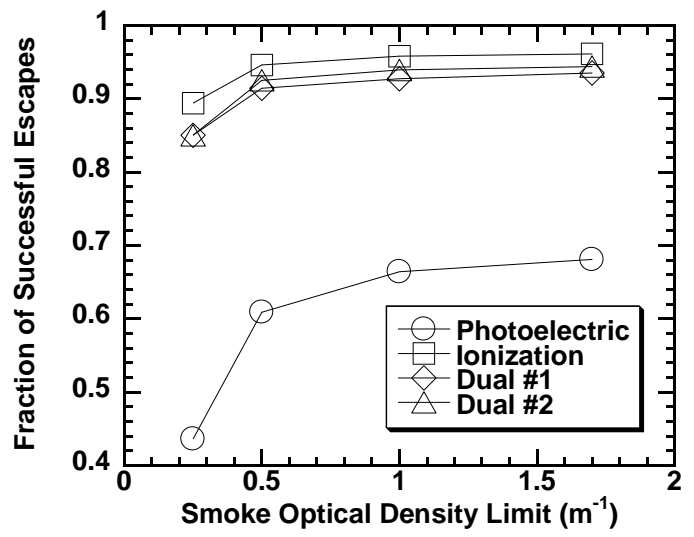

Fig. 10. Fraction of successful escapes $\left(\mathrm{X}_{f}\right)$ for the living room flaming chair fire considering the more vulnerable group.

Figures 11-13 show the fraction of successful escapes for a smoldering fire scenario as a function of alarm type and optical density limit for each frequency distribution. The smoldering fire scenario was an initially smoldering upholstered chair in the living room that transitions to a flaming fire. 


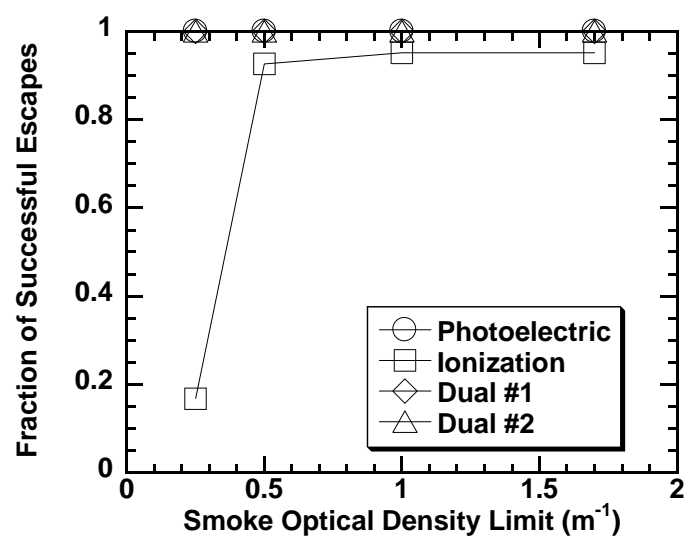

Fig. 11. Fraction of successful escapes $\left(\mathrm{X}_{f}\right)$ for the living room smoldering chair fire considering the easily alerted, mobile group.

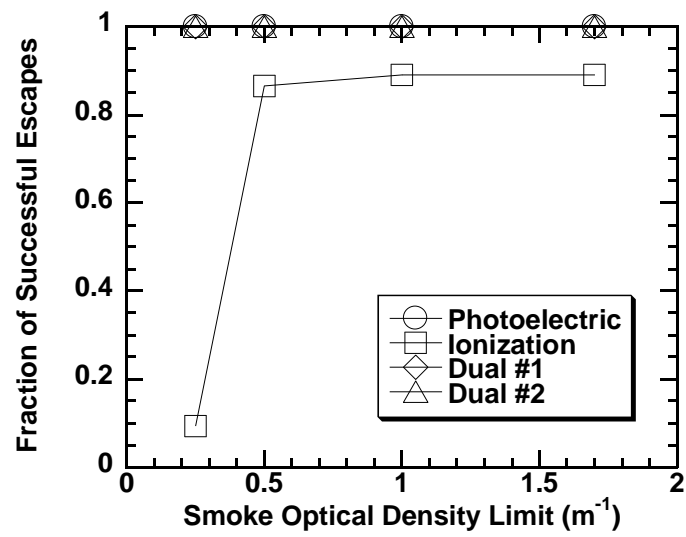

Fig. 12. Fraction of successful escapes $\left(\mathrm{X}_{f}\right)$ for the living room smoldering chair fire considering the moderately mobile group.

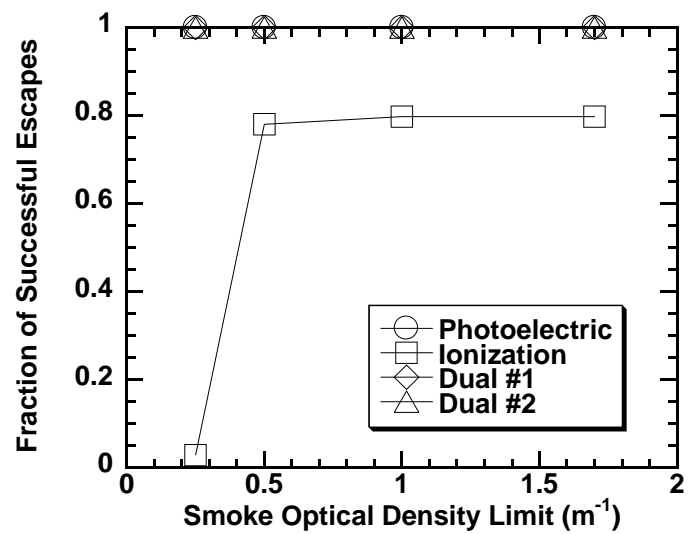

Fig. 13. Fraction of successful escapes $\left(\mathrm{X}_{f}\right)$ for the living room smoldering chair fire considering the more vulnerable group.

For every frequency distribution and optical density limit, the fraction of successful escapes for ionization alarm was lower than photoelectric or dual alarms. This is expected since photoelectric sensors tend to 
alarm sooner to smoldering fires. For every frequency distribution and optical density, the photoelectric and both dual alarms always provided enough time to escape. (Since the frequency distribution is a continuous function that extends to infinity, and the RSET computations only run up to a pre-movement time of $180 \mathrm{~s}$, results were normalized by the cumulative fraction from 0 to $180 \mathrm{~s}$.) There was a very sharp increase in fraction of successful escapes for ionization alarms moving from an optical density limit of $0.25 \mathrm{~m}^{-1}$ to $0.50 \mathrm{~m}^{-1}$, and a much smaller increase up to an optical density limit of $1.7 \mathrm{~m}^{-1}$.

The sensitivity of fraction of successful escapes to the smoke optical density limit observed in the computations above suggests that the combination of accelerating smoke production from flaming fires (or smoldering fires that transitioned to flaming), and the reduction in travel speed conspire to reduce the number of egress scenarios that change from an unsuccessful to successful outcome as the optical density limit was raised. At lower optical density limit values the difference between the fraction of successful escapes for the four alarms are larger, but the ranking trend for $0.25 \mathrm{~m}^{-1}$ and $0.50 \mathrm{~m}^{-1}$ remains the same.

Considering the pre-movement distribution characterizing the slower, more vulnerable population (median value of $55 \mathrm{~s}$ ), the relative effectiveness of photoelectric, ionization, and both dual alarms for all flaming fire scenarios and all smoldering fire scenarios are shown in Figs. 14 and 15 respectively.

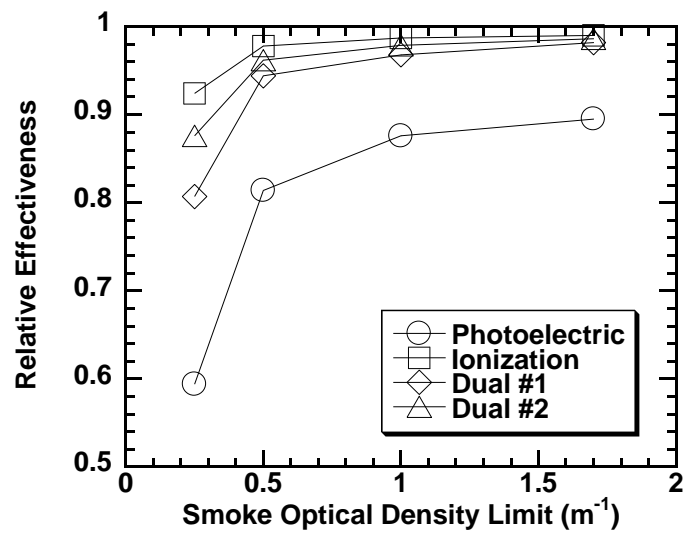

Fig. 14. Relative smoke alarm effectiveness $\left(E_{r}\right)$ averaged over all 12 flaming fire chair tests and all 13 egress scenarios considering the more vulnerable group.

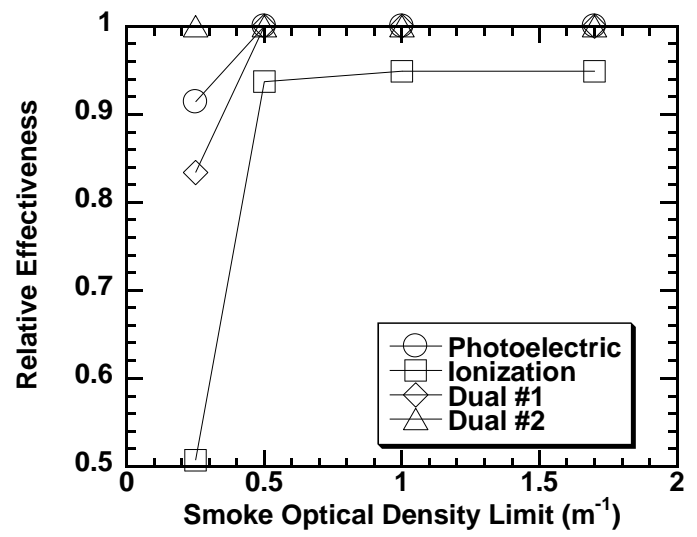

Fig. 15. Relative smoke alarm effectiveness $\left(E_{r}\right)$ averaged over all 12 smoldering chair tests and all 13 egress scenarios considering the more vulnerable group.

For each smoke optical density limit, the photoelectric alarm yielded the lowest average relative effectiveness for flaming fires and the ionization alarm yielded the lowest average relative effectiveness for smoldering fires. There is a steep increase in average relative effectiveness from optical density limits of $0.25 \mathrm{~m}^{-1}$ to $0.50 \mathrm{~m}^{-1}$ for both photoelectric and ionization alarms. The data sets at $0.25 \mathrm{~m}^{-1}$ and $0.50 \mathrm{~m}^{-1}$ 
were subjected to a Tukey test [16] to determine if there were statistically significant differences at a confidence level of $95 \%$ between the average relative effectiveness values for each alarm type. For the flaming fire model results at both smoke optical density limits, there is a statistically significant difference between the photoelectric alarm mean value and all other alarm type mean values, and there is not a statistically significant difference between the ionization alarm mean value and the dual alarm mean values, or between the dual alarm mean values themselves. For the smoldering fire model results, there are only statistically significant differences between the ionization alarm mean value and the photoelectric alarm or dual \#2 alarm mean values at a smoke optical density limit of $0.25 \mathrm{~m}^{-1}$.

While it may be tempting to compute a weighted average alarm effectiveness of initially flaming and smoldering fire scenarios, the fractions of initially flaming and smoldering fires is unknown. Ahrens [17] details US fire statistics for the time period 2003-2007. The percentages of residential fire deaths implicated by various ignition sources were: smoking materials at $25 \%$ (presumably initially smoldering fires), candles at $6 \%$ and playing with heat source at $4 \%$ (both presumably initially flaming fires), heating equipment at $22 \%$, electrical distribution and lighting at $12 \%$, intentional at $12 \%$, and cooking at $17 \%$. An unknown fraction of cooking initiated fires occurs during an un-attended phase where smoke alarms may provide the initial warning. Some fraction of heating equipment, electrical distribution and lighting, intentional, and cooking initiated fires start as flaming fires or initially pyrolyze materials for a short time before transitioning to flaming, and some fraction may initiate a long smoldering phase. While the exact fraction is unknown, both initially flaming and initially smoldering fires are implicated in residential fire deaths; therefore, smoke alarms must provide adequate warning to both flaming and smoldering fires.

The analysis performed here suggests that there is a benefit from a combination of alarm technologies irrespective of the fraction of initially flaming fires. Vulnerable populations who may require significantly more time to escape than more mobile populations would benefit the most from dual alarm technology or side-by-side photoelectric and ionization alarms. In fact, Hall [18] argues that extra escape time could help approximately $1 / 4$ of the fatally and non-fatally injured fire victims in the US (that is, the victims who were attempting escape.) These victims could have benefited from smoke alarm technologies that provided additional escape time.

\section{CONCLUSIONS}

A model was developed that includes a frequency distribution of pre-movement time, and variable travel speed through smoke along with an estimation of tenability conditions in order to quantify the performance of different types of smoke alarms. Analysis of limited pre-movement time studies suggested that a premovement time frequency distribution can be characterized by a log-normal function form. Log-normal fits to three data sets showed that the width was adequately characterized by a geometric standard deviation of 1.6 , even though median values ranged from $9 \mathrm{~s}$ to $55.5 \mathrm{~s}$. The impact of longer escape times due to travel through smoke was included in the model. A performance metric, the relative effectiveness, was proposed to rank the performance of smoke alarms over a range of fire and egress scenarios.

The model was exercised with a data set from the NIST Smoke Alarm Sensitivity Study [15] that included four flaming fire scenarios and four smoldering fire scenarios. Examining the effect of the smoke optical density limit, it was observed that there can be a steep increase in relative effectiveness from a smoke optical density limit of $0.25 \mathrm{~m}^{-1}$ to $0.50 \mathrm{~m}^{-1}$. However, the ranking of smoke alarms tend to remain the same. Relative effectiveness is less sensitive to changes in the optical density limit above $0.50 \mathrm{~m}^{-1}$ because tenability limits due to heat exposure and toxic gases were being reached prior to the optical density limit during lengthy egress scenarios. Photoelectric alarms had the lowest relative effectiveness values for flaming fires, while ionization alarms had the lowest relative effectiveness values for smoldering fires. Given the magnitude of statistically significant mean values of relative effectiveness for all flaming and smoldering fires considered, the model results suggest that there is a benefit from a combination of alarm technologies. Vulnerable populations who may require significantly more time to escape than more mobile populations would benefit the most from dual alarm technology or side-by-side photoelectric and ionization alarms with alarm placement following current NFPA 72 requirements. 


\section{REFERENCES}

[1] Bukowski, R.W., Waterman, T.E., and Christian, W.J., "Detector Sensitivity and Siting Requirements for Dwellings: Report of the NBS Indiana Dunes Tests," No. SPP-43, National Fire Protection Association, Quincy, MA, 1975.

[2] Bukowski, R.W., Peacock, R.D., Averill, J.D., Cleary, T.G., Bryner, N.P., Walton W.D., Reneke, P.A., and Kuligowski, E.D., "Performance of Home Smoke Alarms, Analysis of the Response of Several Available Technologies in Residential Fire Settings," National Institute of Standards and Technology, Tech. Note 1455-1, Gaithersburg, MD, 2008.

[3] Su, J.Z., Crampton, G.P., Carpenter, D.W., McCartney, C., and Leroux, P., “'Kemano Fire Studies - Part 1: Response of Residential Smoke Alarms," National Research Council of Canada, Research Report 108, Ottawa, Canada, 2003.

[4] Cleary, T.G., "Full-scale Residential Smoke Alarm Performance," 14th International Conference on Automatic Fire Detection, University of Duisburg-Essen, Duisburg, Germany, 2009.

[5] NFPA 72-2007, National Fire Alarm Code, National Fire Protection Association, Quincy, MA.

[6] Proulx, G., "Evacuation Time," The SFPE Handbook of Fire Protection Engineering, (4 ${ }^{\text {th }}$ ed) DiNenno P.J. et at. (eds), National Fire Protection Association, Quincy, MA, 2008, p.3/355.

[7] ISO 13571-2007, Life-threatening Components of Fire - Guidelines for the Estimation of Time Available for Escape Using Fire Data, International Organization for Standardization, Geneva.

[8] Jin, T., and Yamada, T., (1985) Irritating Effects of Fire Smoke on Visibility, Fire Science and Technology 5(1): 79-90, http://dx.doi.org/10.3210/fst.5.79

[9] Nober, E., Peirce, H., and Well, A., "Waking Effectiveness of Household Smoke and Fire Detection Devices," National Bureau of Standards Report NBS-GCR 83-439, Gaithersburg, MD,1983.

[10] Proulx, G., Cavan, N., and Tonikian, R., "Egress Times from Single Family Houses," Institute for Research in Construction, National Research Council Canada, Research Report:IRC-RR-209, Ottawa, Canada, 2006.

[11] Purser, D.A., (2003) ASET and RSET: Addressing Some Issues in Relation to Occupant Behavior and Tenability, Fire Safety Science 7: 91-102, http://dx.doi.org/10.3801/IAFSS.FSS.7-91

[12] Duncan, C., "The Effectiveness of the Domestic Smoke Alarm Signal," School of Engineering, University of Canterbury, Fire Engineering Research Report 99/5, Christchurch, NZ, 1999.

[13] Akizuki, Y., Tanaka, T., and Yamao, K., (2009) Calculation Model for Travel Speed and Psychological State in Escape Routes Considering Luminous Condition, Smoke Density and Evacuee's Visual Acuity, Fire Safety Science 9:365-376, http://dx.doi.org/10.3801/IAFSS.FSS.9$\underline{365}$

[14] Gwynne, S., and Rosenbaum, E., "Employing the Hydraulic Model in Assessing Emergency Movement," The SFPE Handbook of Fire Protection Engineering, $\left(4^{\text {th }}\right.$ ed $)$, DiNenno P.J. et al. (eds), National Fire Protection Association, Quincy, MA, 2008, p3/373.

[15] Cleary, T.G., (2010) Results from a Full-Scale Smoke Alarm Sensitivity Study, Fire Technology, http://dx.doi.org/10.1007/s10694-010-0152-2

[16] NIST/SEMATECH e-Handbook of Statistical Methods, Croarkin, C., and Tobias, P. (eds), National Institute of Standards and Technology, Gaithersburg, MD, 2010.

[17] Ahrens, M., "Home Structure Fires," National Fire protection Association, Quincy, MA, 2010.

[18] Hall, J., (2004) How Many People Can be Saved from Home Fires if Given More Time to Escape, Fire Technology 40: 117-126, http://dx.doi.org/10.1023/B:FIRE.0000016839.11376.b3 